\title{
Italian experiences in the management of andrological patients at the time of Coronavirus pandemic
}

\author{
Carlo Maretti ${ }^{1}$, Andrea Fabiani ${ }^{2}$, Fulvio Colombo ${ }^{3}$, Alessandro Franceschelli ${ }^{3}$, Giorgio Gentile ${ }^{3}$, \\ Franco Palmisano ${ }^{3}$, Valerio Vagnoni ${ }^{3}$, Luigi Quaresima ${ }^{4}$, Massimo Polito ${ }^{5}$ \\ ${ }^{1}$ Department of Andrology, CIRM Medical Center, Piacenza, Italy; \\ ${ }^{2}$ Unit of Urology, Surgical Department, Macerata Hospital, Area Vasta 3, ASUR Marche, Macerata, Italy; \\ ${ }^{3}$ Andrology Unit, University Hospital S.Orsola-Malpighi, Bologna, Italy; \\ ${ }^{4}$ Urology Division at the Civitanova Marche Hospital, Civitanova Marche, Italy; \\ ${ }^{5}$ Department of Clinical and Specialist Sciences, Division of Urology, Polytechnic University of the Marche Region Medical School, \\ Ancona, Italy.
}

\begin{abstract}
Summary
The SARS-CoV-2 (Severe acute respiratory syndrome coronavirus 2) was first reported in December 2019, then its rapid spread around the world caused a global pandemic in March 2020 recording a high death rate. The epicenter of the victims moved from Asia to Europe and then to the United States. In this Pandemic, the different governance mechanisms adopted by local health regional authorities made the difference in terms of contagiousness and mortality together with a community strong solidarity. This document analyzes the andrological urgencies management in public hospitals and in private practice observed in Italy and in particular in two of the most affected Italian Regions: Emilia-Romagna and Marche.
\end{abstract}

KeY WORDS: SARS-CoV-2; Pandemic; Andrology; Public hospitals; Private practice.

Submitted 9 January 2021; Accepted 21 January 2021

\section{INTRODUCTION}

The International Committee on Taxonomy of Viruses (ICTV), responsible for defining the official classification of viruses and the taxonomy of the Coronaviridae family, has officially classified under the name SARS-CoV-2 (Severe acute respiratory syndrome coronavirus 2 ) the virus provisionally called by the World Health Organization (WHO) 2019-nCoV and responsible for COVID-19 cases ("CO" stands for corona, "VI" for virus, "D" for disease and "19" indicates the year in which it occurred) (1-3).

SARS-CoV-2 was identified for the first time in Wuhan, in the province of Hubei in China. Italy was the first country outside of China involved by pandemic. The Italian Council of Ministers declared the state of emergency from 31.01.2020. The Italian Prime Minister extended some restrictive measures concerning gatherings and movements throughout the national territory with effect from $10^{\text {th }}$ March. This logic of restrictions was above all to reduce the contagiousness (RO) of COVID-19. The WHO declared the world pandemic on 11 March 2020 (4).

WHO had warned each country of the risk of the "tsunami" of information, in particular fake news would have led to panic situations (supermarket raids, unnecessary visits to hospital emergency rooms, uncontrolled depar- tures to other places, riots in prisons, etc.). At the declaration of a pandemic, the WHO added the risk of an "Infodemia", that is the dissemination of a considerable amount of information, coming from different and often unverifiable sources. Providing the correct information would be an important issue to help and reduce contagiousness and so mortality.

SARS-CoV-2 has widely spread in less than three months because of a globally interconnected world. SARS-Cov-2 appears to spare children while older population with concomitant morbidity are severely affected, in particular males $(5,6)$.

The symptomatic picture includes various symptoms according to the evolution of the disease. The symptoms most commonly observed in patients before hospitalization may be fever, chills, dry cough, dyspnea, asthenia, myalgias and/or arthralgias, while nausea and vomit, nasal congestion, hemoptysis, diarrhea, conjunctival congestion are less common (7).

This symptomatology can be complicated starting from the third/fourth week, often in a dramatically increasing manner such as to require hospitalization. In severe cases, pneumonia, acute respiratory distress syndrome, sepsis and septic shock, endothelial dysfunction with thromboembolism can occur till to the patient's death (8).

It is important to remember that the patient with SARSCoV-2 can also be asymptomatic or pauci-symptomatic, thus contributing to the spread of the virus in the community (9). In humans, the transmission pathways of the COVID-19 virus are mainly direct, that is, through the respiratory tract with the inhalation of respiratory droplets that are generated when an infected person speaks, coughs or sneezes. Viral transmission can also be indirect, i.e. mediated by inanimate vectors (soil, personal effects, paper sheets, money, plastic or metal surfaces, etc.) and in any case it will depend on the viral load present on that surface. Relatively to the potential COVID-19 sexual transmission, there are no definitive data. The impact on male fertility and the potential of the spermatozoa to serve as vectors for the sexual transmission of this disease are not clear yet, although some biological characteristics of spermatozoa must leave researchers open to these possi-

No conflict of interest declared. 
bilities $(10,11)$. This document analyzes the various experiences of Italian uro-andrologists in their hospital wards as well as in andrological private urgencies in EmiliaRomagna and Marche, in this lockdown period.

\section{EXPERIENCES IN ANDROLOGY MANAGEMENT in ItALIAN CENTERS DURING COVID-19 PANDEMIC}

\section{Department of Andrology, CIRM Medical Center, Piacenza}

From 30.01.2020 when the Italian Council of Ministers declared a state of health emergency, it was decided to study in details the dynamics transmission of COVID-19 infection, trying to make a critical review of the scientific literature data in order to better manage a possible state of emergency. The perception of an individual threat, is the fear that triggers some physiological reaction of anxiety, which can also generate sexual troubles (erectile dysfunction, for example). Working in the private sector of andrology, it was tried to protect patients and personnel adopting several precautions to limit the contagion (disposable surgical masks, shoe covers, gloves, aprons, headgears and visors). Only urgent visits were scheduled and telematic advice was produced in required cases. On the first days of lockdown there was not any request for andrological visit and the ones previously booked were cancelled by the patients themselves. After about ten days, people with a probable erectile dysfunction of stressogenic nature, began to call for telematic suggestions. It was clear that confinement, the loss of the usual routine, and the reduction of social relationships had increased the sense of insecurity and anguish $(12,13)$. During the same period most of the urgent visits to males were related to infections (testicular, prostatic, urethral, glans or preputial), probably related to an increased frequency of sexual acts during the lockdown period. The patients were received individually, equipped with disposable PPE (Personal Protective Equipment, the Italian DPI ) waiting alone for their turn. No visits for couple infertility were performed, according to the indication claimed by National Health Authorities regarding the need to stop the search for children.

\section{Uro-Andrologic Unit, Policlinico di S.Orsola, Bologna}

From March 10, due to the measures introduced to limit the spread of the COVID-19, our academic hospital established urgent measures to reduce outpatient procedures, use of operating theatres and non-urgent clinical activity. Initially, the Uro-Andrologic Department has been turned into medical department due to need for the management and treatment of patients with SARS-CoV2; then, Urological procedures were referred to another Centre with limited number of beds, reduction of operating theatres (from four to one per day) and priority for malignancy and obstructive uropathy conditions while andrological activities has been dramatically cancelled except for one case of three-components penile prosthesis reservoir removal for confirmed local infection. To date, no malignancies or other urgent andrological procedures were scheduled during the restriction. In this scenario, the staff of Urology and Andrology Department has been used to enhance assistance in the medical departments in order to support the activity of Internists. Outpatient activities were limited to urgent consultations while patients with non-urgent (whenever possible consulted by telephone) have been postponed, pending provisions relating to the end of the lockdown.

The psychological impact of the COVID-19 outbreak among the whole and specifically andrological patients is still unknown. As expected, our web and Facebook pages counted an increase in visualization as well as an increasing in requests regarding access to outpatient consultation. Interestingly, most of the patients reported conditions related to psychological burden such as penile enlargement and erectile dysfunction surgery.

\section{Department of Surgery, Section of Urology, Macerata Hospital}

In this public community hospital in the Marche Region, the shift from patient centered medicine to a community centered approach was immediate. The cancellation of the scheduled operating sessions was the consequence of the need to make surgical decision no longer based on individual patients' needs but on the availability of beds in intensive and sub-intensive care for the management of patients suffering from SARS-CoV-2, mainly from neighboring areas of the northern part of the Region. The immediate cancellation meant the suspension of the surgical treatment of the non-oncological pathologies. In this situation, the andrological surgery, in its vast part, has suffered a drastic stop. Circumcisions, Peyronie's disease or congenital recurvatum surgeries, varicocele and infertility surgeries, surgical treatment of erectile dysfunction have been cancelled. Urology Division in Macerata Hospital has a 10-bed ward and, normally, 6 full time urologists and 1 resident of the Marche Polytechnic University: before Covid pandemic about 1000 surgical procedures and 4500 visits per year were performed. Our attitude was prompted to define our surgical and outpatient activities by following the provisions elaborated by the reference scientific societies (13-16). However, the feeling was to be neglected by the medical direction regarding priorities in the planning of procedures. It was difficult to obtain anesthesiologist assistance in non- oncological urological procedures (for example: ureteral stenting). The feeling was that it was forbidden to have pathology other than SARS-CoV-2.

Urology Division was managed as a mixed ward hosting non-COVID and COVID patients who did not require intensive care support. One Urologist per week was commanded to participate at medical activities, alone or alongside with Internal Medicine colleagues.

Consequently, from the beginning of the lockdown, the andrological activity was reduced to the management of several cases with complications such as phimosis whose surgery had been postponed, one spermatic cord torsion (Figure 1), two testicular neoplasms, metastatic priapism (two cases), one penile cancer (Figure 2) and three cases of Mondor's disease (Figure 3).

Requests for advice in relation to non-acute testicular pain, even by telephone, were increased.

There have been no cases of penile trauma which had been unusually more frequent in the 6 months preceding the pandemic. 


\section{Figure 1.}

Spermatic cord torsion. Pre- (left) and post (right)-derotation testicle aspect.
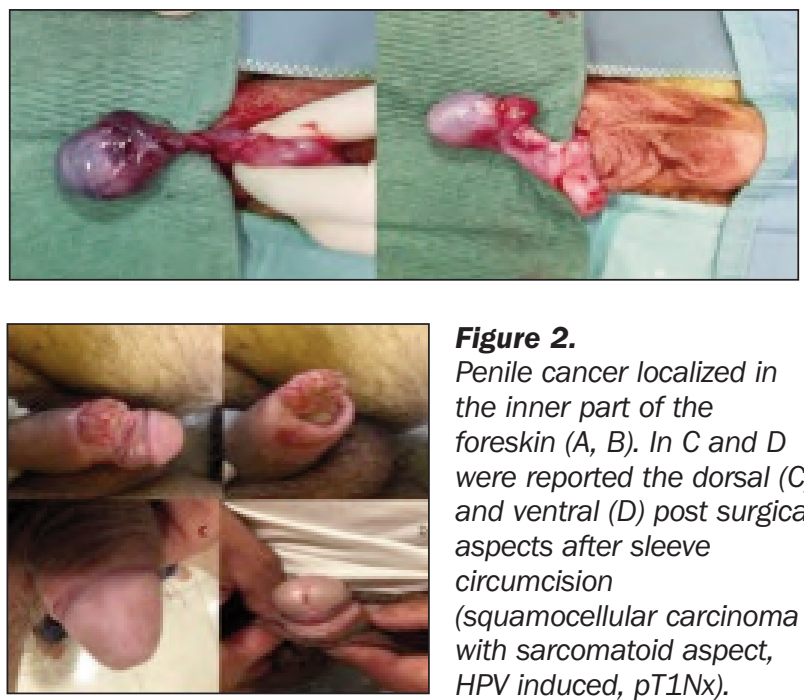

Figure 2.

Penile cancer localized in the inner part of the foreskin ( $A, B)$. In $C$ and $D$ were reported the dorsal $(C)$ and ventral $(D)$ post surgical aspects after sleeve circumcision

(squamocellular carcinoma with sarcomatoid aspect, HPV induced, $p T 1 N x)$.

\section{Figure 3.}

Mondor disease. Clinical aspect (left) in a circumcised penis. Ultrasound features (right) with thrombus (white star) occluding the dorsal superficial vein lumen.

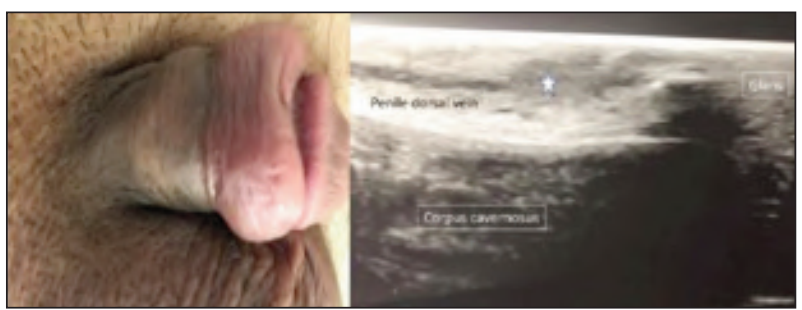

\section{U.O.S. Urologic Surgery, Azienda Ospedali Riuniti, Ancona}

The Marche Region was the $5^{\text {th }}$ Italian Region in terms of numbers of patients, hospitalized and deaths. The Ospedali Riuniti in Ancona, which play the role of Regional Referral Centre (Hub), immediately gave its orders to convert surgical beds, thus limiting all the other surgical activity, in order to grant, for resuscitation purposes, and therefore to provide physician availability for emergency and non-intensive medical unit for Covid. Eight Unit dedicated to Covid were established in a few weeks. The elective Andrological activity was interrupted. The urological ward was reduced to 15 of 22 beds and a waiting room ( 2 beds) was dedicated to patients admitted from emergency waiting the result of Covid-test analysis. Surgery was planned only for emergency/urgency, symptomatic tumors, kidney failure, infection, diseases with ongoing complications and traumatic events. Urological and Andrological outpatient activity, diagnostic ultrasound and cystoscopy, and endovesical chemo or BCG were temporary closed.

To date, the lockdown throughout the country has greatly reduced the incidence of traumatic events related to road traffic and work activities. In the first 50 days we had to intervene on two traumas of the genital tract that happened due to working activities. We listed criteria to plan andrological surgery in agreement with our experience, the Covid Task Force Unit in our hospital and published
Guidelines on urological surgery (17-21). The andrological surgical activity included two orchiectomies for testicular cancer, one penile amputation with inguinal and ipsilateral pelvic lymph node dissection for penile cancer (Figure 4) and one surgery for severe gangrenous necrotizing fasciitis of the genitals in a 58-year male with diabetes, HIV and drugs addiction. This case resolved after 75 days (Figure 5). The overall surgical activity was reduced by $47 \%$ with 262 procedures from March $1^{\text {st }}$ to May $30^{\text {th }}$ 2020 compared to 492 during the same time period in 2019. However the Urology resident training was guaranteed all the time also supported by web lessons (22).

\section{Urology Division at the Civitanova Marche Hospital}

As soon as the pandemic from Coronavirus emerged, the community Hospital in Civitanova Marche was been converted into a Covid-Hospital, as well as most of the staff who were employed in it. All departments were progressively occupied by symptomatic COVID-19 positive patients, diverting patients affected by other pathologies to other hospitals (Macerata, Ancona) and suspending the outpatient and surgical activity. From March 24, 2020, urologists started to work as an internist-type ward in shifts of medium-low care intensity, with a shift of 8

\section{Figure 4.}

Simultaneous right inguinal and pelvic lymph node dissection through modified inguinal incision (Surgical Andrology Unit, Ospedali Riuniti, Ancona).

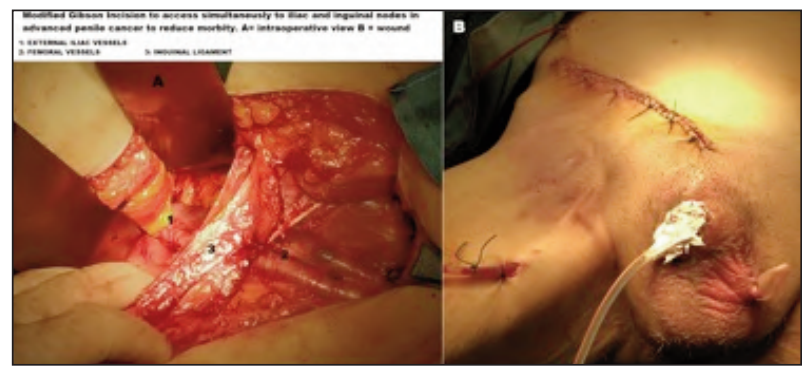

\section{Figure 5.}

Genitalia gangrene management (Surgical Andology Unit, Ospedali Riuniti, Ancona).

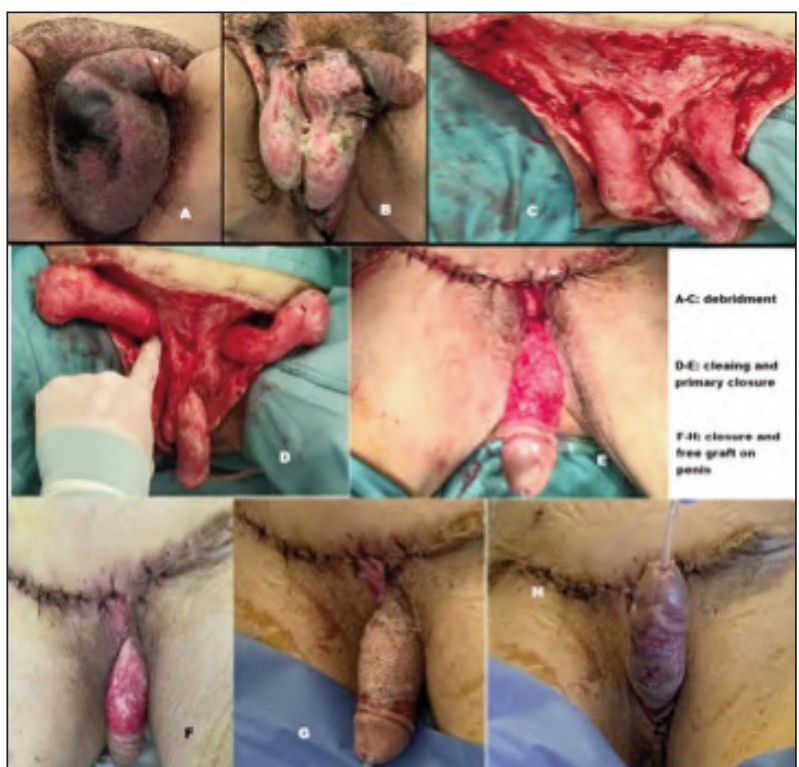


hours a day. During their shifts, urologists were also called to perform urological consultations for positive Covid-19 patients hospitalized in the various wards of the hospital; during this activity in the hospital, there was no request for some andrological advice.

In addition to the hospital activity, private Andrologist activity was continued by activating an online consultancy function with video consultations: this activity has proven to be safe, effective and pleasing to the patients who have used it. During this activity, five requests for advice for erectile deficit mainly of recent onset were received. The cause of these deficits is often psychogenic, probably linked to the state of prostration, inhibition related to social distancing and quarantine. Therefore, it is important not to increase the patient's concern or treat him as an organic erectile deficit, but to deepen the diagnosis if it persists even after the lockdown phase.

\section{Conclusions}

Males and andrology community are still paying the price of a delay in the management of the pandemic, fueled by first conflicting messages and fake news. The key-role of andrologists in Italy was to offer themselves to support COVID patients and support andrological patients seeking help by web, phone and email. Andrological surgery is still restricted in most hospitals and, especially in public setting, nevertheless how and when re-schedule suppressed operations is missing yet. The lack of epidemiological data on andrological pathologies in the lockdown period stimulated us to expose our experiences which, in addition to an interruption of the specialist activity in hospital structures, highlighted above all the andrological pathologies that had been managed in a private setting.

\section{References}

1. Lefkowitz EJ, Dempsey DM, Hendrickson RC, et al. Virus taxonomy: the database of the International Committee on Taxonomy of Viruses (ICTV). Nucleic Acids Res. 2018; 46:D708-D717.

2. Coronaviridae Study Group of the International Committee on Taxonomy of Viruses. The species Severe acute respiratory syndrome-related coronavirus: classifying 2019-nCoV and naming it SARS-CoV-2. Nat Microbiol. 2020; 5:536-544.

3. Song Z, Xu Y, Bao L, et al. From SARS to MERS, thrusting coronaviruses into the spotlight. Viruses. 2019; 11:59.

4. Decrete of the president of the council of ministers, Italy, 8 March 2020. OJ No. 59 of 8-3-2020

5. Wölfel R, Corman VM, Guggemos W, et al. Virological assessment of hospitalized patients with COVID2019. Nature. 2020; 581:465469 .

6. Hui DSC, Zumla A. Severe acute respiratory syndrome: historical, epidemiologic, and clinical features. Infect Dis Clin North Am. 2019; 33:869-889.

7. Huang C, Wang Y, Li X, et al. Clinical features of patients infected with 2019 novel coronavirus in Wuhan, China. Lancet. 2020; 395:497-506.

8. Jin Y, Yang H, Ji W, et al. Virology, epidemiology, pathogenesis, and control of COVID-19. Viruses. 2020; 12:372.

9. Borges do Nascimento IJ, Cacic N, Abdulazeem HM, et al. Novel coronavirus infection (COVID-19) in humans: a scoping review and meta-analysis. J Clin Med. 2020; 9:941.

10. Aitken RJ. COVID-19 and human spermatozoa-Potential risks for infertility and sexual transmission? Andrology. $2020 \mathrm{Jul}$ 10:10.1111/andr.12859.

11. Maretti C, Privitera S, Arcaniolo D, et al. COVID-19 pandemic and its implications on sexual life: Recommendations from the Italian Society of Andrology. Arch Ital Urol Androl. 2020; 92:73-77.

12. Brooks SK, Webster RK, Smith, LE, et al. The psychological impact of quarantine and how to reduce it: rapid review of the evidence. Lancet. 2020; 395:912.

13. Leonardi R, Bellinzoni P, Broglia L, et al. Hospital care in Departments defined as COVID-free: A proposal for a safe hospitalization protecting healthcare professionals and patients not affected by COVID-19. Arch Ital Urol Androl. 2020 Apr 24; 92:67-72.

14. Simonato A, Giannarini G, Abrate A, et al. Pathways for Urology patients during the Covid19 pandemic. Minerva Urol Nefrol. 2020; 72:376-383.

15. Ficarra $V$, Novara $G$, Abrate A, et al. Urology practice during COVID-19 pandemic. Minerva Urol Nefrol. 2020; 72:369-375.

16. Stensland KD, Morgan TM, Moinzadeh A, et al. Considerations in the triage of urologic surgeries during the COVID-19 pandemic. Eur Urol. 2020; 77:663-666.

17. Martino P, Galosi AB, Bitelli M, et al. Imaging Working GroupSocieta Italiana Urologia (SIU); Società Italiana Ecografia Urologica Andrologica Nefrologica (SIEUN). Practical recommendations for performing ultrasound scanning in the urological and andrological fields. Arch Ital Urol Androl. 2014; 86:56-78.

18. Maselli G, Cordari M, Catanzariti F, et al. Penile Gangrene by Calciphylaxis: An Unusual Clinical Presentation in a Patient with Diabetic Nephropathy on Hemodialysis. J Emerg Med 2017; 52:e255e256.

19. Galosi AB, Capretti C, Leone L, et al. Pseudoaneurysm with arteriovenous fistula of the prostate after pelvic trauma: Ultrasound imaging. Arch Ital Urol Androl. 2016; 88:317-319.

20. Dell'Atti L, Cantoro D, Maselli G, Galosi AB. Distant subcutaneous spreading of Fournier's gangrene: An unusual clinical identification by preoperative ultrasound study. Arch Ital Urol Androl. 2017; 89:238-239.

21. Dell'Atti L, Scarcella S, Tallè M, et al. Simultaneous curvature correction at the time of the penile fracture repair: surgical and functional outcomes. Res Rep Urol. 2019; 11:105-110.

22. Busetto GM, Del Giudice F, Mari A, et al. How Can the COVID19 Pandemic Lead to Positive Changes in Urology Residency? Front Surg. 2020; 7:563006.

\section{Correspondence}

Carlo Maretti, MD - carlomaretti@tin.it

Department of Andrology, CIRM Medical Center, Piacenza, Italy

Andrea Fabiani, MD - andreadoc1@libero.it

Unit of Urology, Surgical Department, Macerata Hospital, Area Vasta 3, ASUR Marche, Macerata, Italy

Fulvio Colombo, MD - fulvio.colombo@aosp.bo.it

Alessandro Franceschelli, MD - alessandrofranceschelli@yahoo.it

Giorgio Gentile, MD - dr.giorgio.gentile@gmail.com

Franco Palmisano, MD - franco.palmisano@hotmail.it

Valerio Vagnoni, MD - vagnoni.dr@gmail.com

Andrology Unit, University Hospital S.Orsola-Malpighi, Bologna, Italy

Luigi Quaresima, MD - luigiquaresima@yahoo.it

Urology Division at the Civitanova Marche Hospital, Civitanova Marche, Italy

Massimo Polito, MD - max_polito@virgilio.it

Department of Clinical and Specialist Sciences, Division of Urology, Polytechnic

University of the Marche Region Medical School, Ancona, Italy 